\title{
Development of Pallet Recognition System using Kinect Camera
}

\author{
Ji-Youn Oh, Hyeung-Sik Choi, Sung-Hun Jung, Hwan-Seong Kim \\ and Hee-Young Shin
}

Korea Maritime and Ocean University, 727 Taejong-ro, Yeongdo-Gu, Busan 606-791, South Korea pellicmeriz@naver.com,hchoi@kmou.ac.kr,director@kmou.ac.kr, \• hskim@kmou.ac.kr,vakarin@naver.com

\begin{abstract}
This study proposes a new pallet recognition system nising Kinect camera. Depth image of Kinect camera is produced from the infrared ray data of random dot type. This system was applied to an automated guided vehicle $(A G V)$ to recognize the pallet in various conditions. A modularized hardware and software of the pollet recognition system was developed. The performance of the developed pallet recognit $10 n$ system was tested through experiments under various environment, and it show good performance.
\end{abstract}

Keywords: Depth Image, Kinęt Camera, Pallet Récognition System, Automated Guided Vehicle $(A G V)$, Unmanned Forklift

\section{Introduction}

The number and scope of logistics centers are showing an increasing trend along with recent advances in $10 \mathrm{~g}$ stics industry. Moreover, work types are continuously varying in distribution centers, and the types of cargo volume and freight are also increasing. For those reasons, the introduction of logistics center automation system is crucial. The pallet recognition system,(PRS) of automatic guided vehicle (AGV) is the core technology in automation system to keep pace with the rapid changes in the logistics centers, and related studies are in progress.

The back projection-based pallet recognition system extracts pallet areas considering the fact that the shade of holes on the pallet surface is darker than that of other pallet areas and the standardized information of pallet, despite changes in color, illumination and background. However, creating the algorithms for this system is considerably more difficult and complex [1]. Recognition system using a laser rangefinder requires expensive instrumentation making its installation, management and maintenance more complex [2]. Stereo camera with the PRS is able to measure the pallet motion and position without precision calibration process, but unable to measure the pallet at a high position unless a special marking is given [3].

This study was performed to develop a new pallet recognition system using the depth image of Kinect camera robust to changes in environment and noise to resolve technical limitations of previous studies. The performance of the developed PRS was tested through experiments. 


\section{Composition of Pallet Recognition System}

This study proposes the PRS that operates based on depth image of Kinect camera and verifies recognition function with robust adaptation to different conditions and noise. Recognition system is integrated to achieve these goals. Depth image of Kinect camera realizes image by receiving numerously scattered or reflected radiations from the infrared ray data of random dot type as shown in Figure 1. Short-distance is expressed in bright color and long-distance is expressed in dark color. The use of infrared ray data facilitates the system to recognize pallets under a wide range of environments. The study aims to realize depth image utilizing the characteristics of Kinect camera.

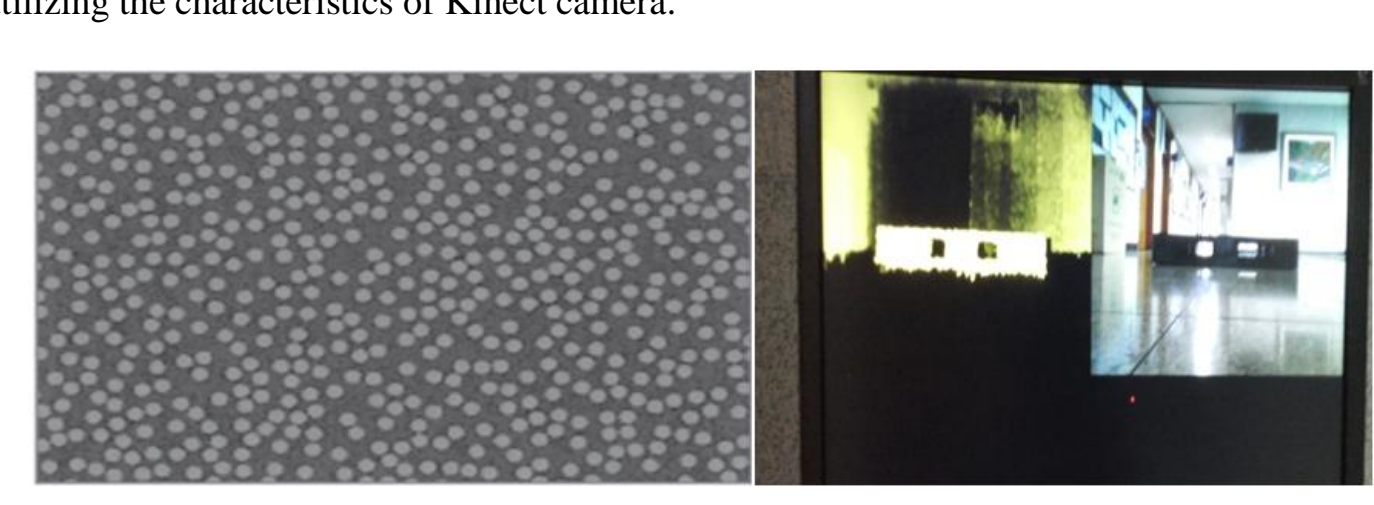

Figure 1. Random dot pattern(Left) Fand Comparison of Depth and RGB(Right)

\subsection{Hardware Development}

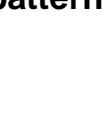

The pallet recognition system uses industriral embedded PC to efficiently transmit and receive depth image data and adequately process imagery information at the same time as shown in Figure 2. Moreover, the system is modularized to be applicable to the actual industrial fields using high-speed SSD (solid state drive) to promptly receive and save imagery data.

\subsection{Software Development}

User interface program is responsible for communication of recognized data including imagers transmission/reception, port designation, and setting of recognition parameters. This program is created to set up camera recognition screen and menus items for various parameter options in the upper part, and composed of recognition time shift, communication port, and other options for menu setting in the lower part as shown Figure 2. Thus, user can set up direct recognition and various parameter options.

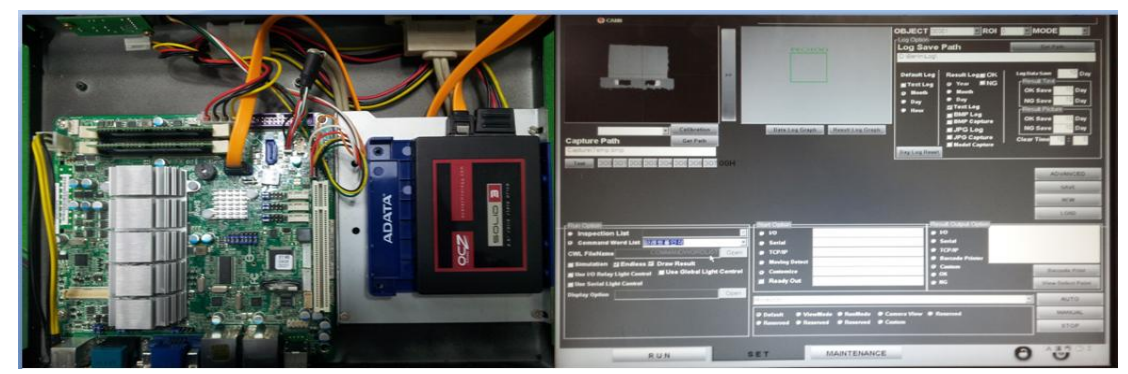

Figure 2. Hardware System(Left) and Software(Right) 
Home screen is designed to display a screen of pallet recognition and inform whether pallet is recognized or not for users to easily identify pallet recognition. Detailed options of user interface program comprises advanced menu items to process labeling, threshold, and calibration of images, set up data ports, and alter recognition time.

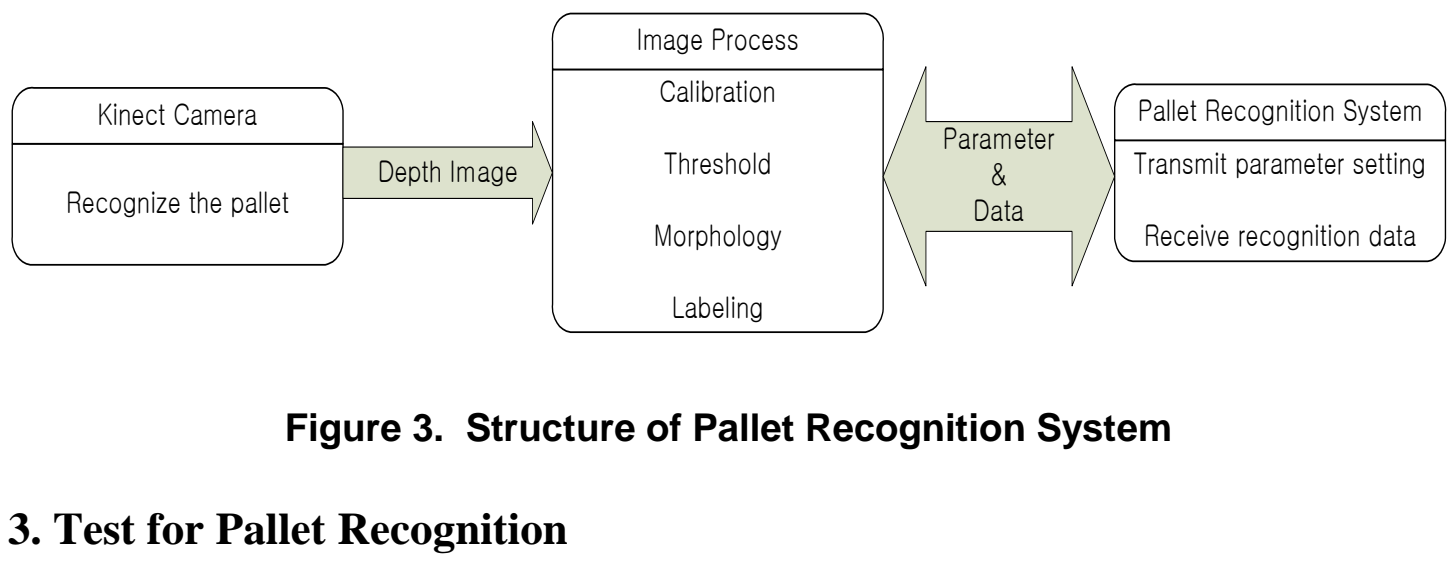

This study developed the PRS to recognize the front side of pallet using hardware system devised to utilize the depth image recognition characteristics of Kinect camera. When a forklift inserts the forks into a pallet loaded with freight, the pallet surface facing forklift carriage should be parallel to Kinecteamera. Therefore, the test was performed in a state of Kinect camera and pallet facingeach other.

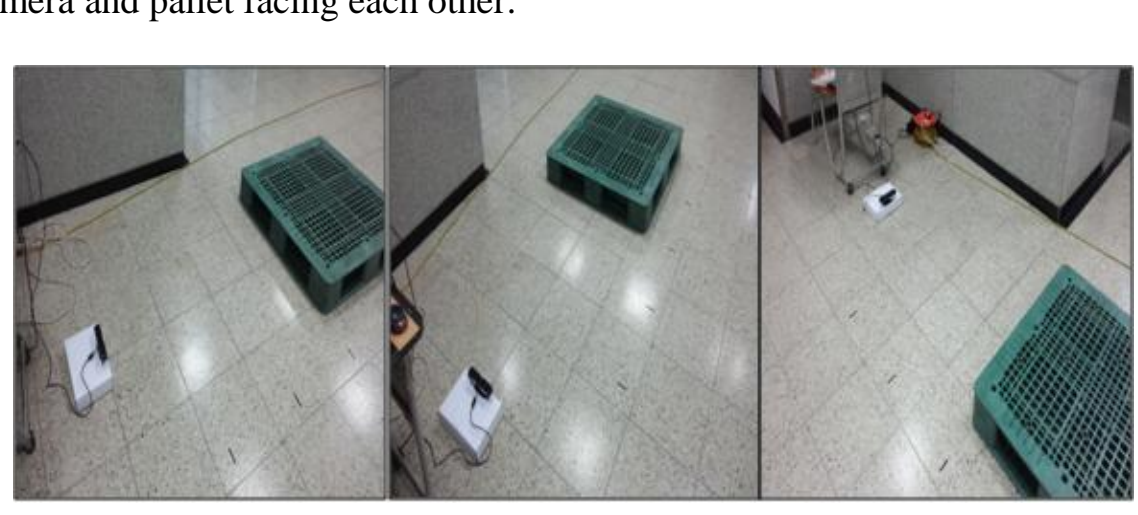

Figure 4. Environment for test of PRS

\subsection{Environment for Pallet Recognition Test}

Recognition rate varies depending on the installed location of pallet recognition system on automatic guided vehicle. Therefore, the installation location of camera has to be taken into account. When a camera is attached to on the top of a forklift, errors may be incurred in recognition due to the bright and dark regions of pallet holes. When a camera is attached to the bottom of a forklift, the camera lens's angle-of-view could be affected and recognition rate could not be obtained. For those reasons, the installation location of camera was decided in the middle of the AGV's forklift carriage in the study. The experiment was conducted with a camera located in a similar height of the premise as shown in Figure 3. Illustrates a standard plastic pallet used in the test.

A range of recognition distance $(0.8 \sim 3 \mathrm{~m})$ of Kinect camera and the length of the forklift forks were taken into consideration. Minimum recognition distance was defined 
as $1.5 \mathrm{~m}$ since more than $1.5 \mathrm{~m}$ of extra recognition distance from camera location was essential. Maximum recognition distance was limited to $2.5 \mathrm{~m}$ because the recognition rate of Kinect camera could be reduced if the distance is too far. Moreover, tests were repeatedly performed under different settings of standard, dark, and freight loaded conditions to verify the recognition capability in a variety of settings.

Table 1. Result of Pallet Recognition

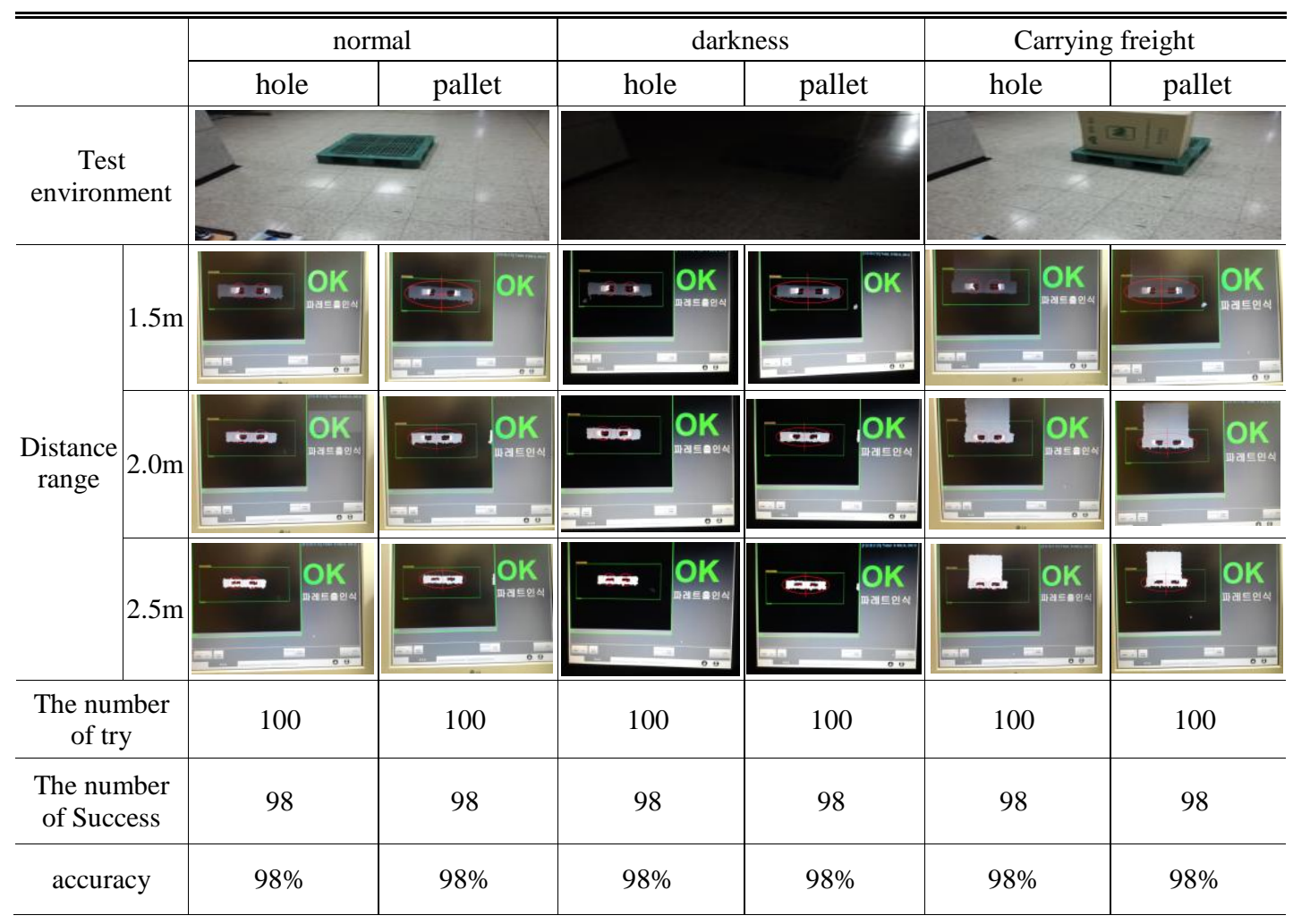

\subsection{Result of the Test}

Tests were carried out under normal, dark, and freight carrying environments at distances of $1.5 \mathrm{~m}, 2.0 \mathrm{~m}$, and $2.5 \mathrm{~m}$ by taking a range of recognition distance of Kinect camera intogceount. Test results are presented in the Table below. Recognition tests were diyided into two recognition points of an entire pallet and pallet holes according to each distance and setting. The recognition rates drawn from the experiment were chosen based on the number of successes when a total of 100 attempts were made by checking accurate recognition of the pallet and pallet holes.

Table 1 shows the results of the experiment for recognition of pallet holes and pallet in normal, dark, and freight loaded settings according to distances. Since depth image is imagery information of infrared ray data, recognition rate achieved was $98 \%$ regardless of brightness of surrounding environment. The system was identified to be firmly adapted to different settings by exhibiting a recognition rate of $98 \%$ in freight carrying environment. In the experiment for pallet recognition, the system recognized the pallet by robustly adapting to varying environments like the results of pallet holes and pallet recognition. 


\section{Conclusion}

This study proposes a new pallet recognition system using depth image of Kinect camera and the performance of the developed system was tested through experiments under various conditions. The PRS is designed to recognize pallets and robustly adapt to rapidly changing complex logistics centers. To apply the system to the actual industrial fields, the system was modularized using industrial embedded computer and user interface system was established for users to set up and control parameters themselves. Tests were performed under normal, dark, and freight loaded environments according to different distances of $1.5 \mathrm{~m}, 2.0 \mathrm{~m}$ and $2.5 \mathrm{~m}$, considering a range of recognition distance of Kinect camera. Recognition rate was $98 \%$ in both pallet and pallet holes recognition.

\section{Acknowledgements}

This research was supported by a grant(11Transportation System - Logistics02) from Transportation System Efficiency Program funded by Ministry of Land, Infrastructure and Transport of Korean government.

\section{References}

[1] S. M. Byun and M. H. Kim, "Pallet Measurement Meth»d for Automatic Pallet Engaging in Real-Time", Journal of Korea Multimedia Society, vol 14. (2011), pp. 171-181.

[2] J. J. Park and S. S. Kim, "Pallet Recognifion of Forklit AGV Using Laser Range Finder", Conference of the ICROS \& KROS, (2009) December, pp. 101-103.

[3] K. D. Hong, A. Brydon and J. D. Seelinger, "Yoder. Automatic Visual Guidance of a Forklift Engaging a Pallet", Robotics and Autonomgus Systems, vp1 54, (2006), pp.1024-1038.
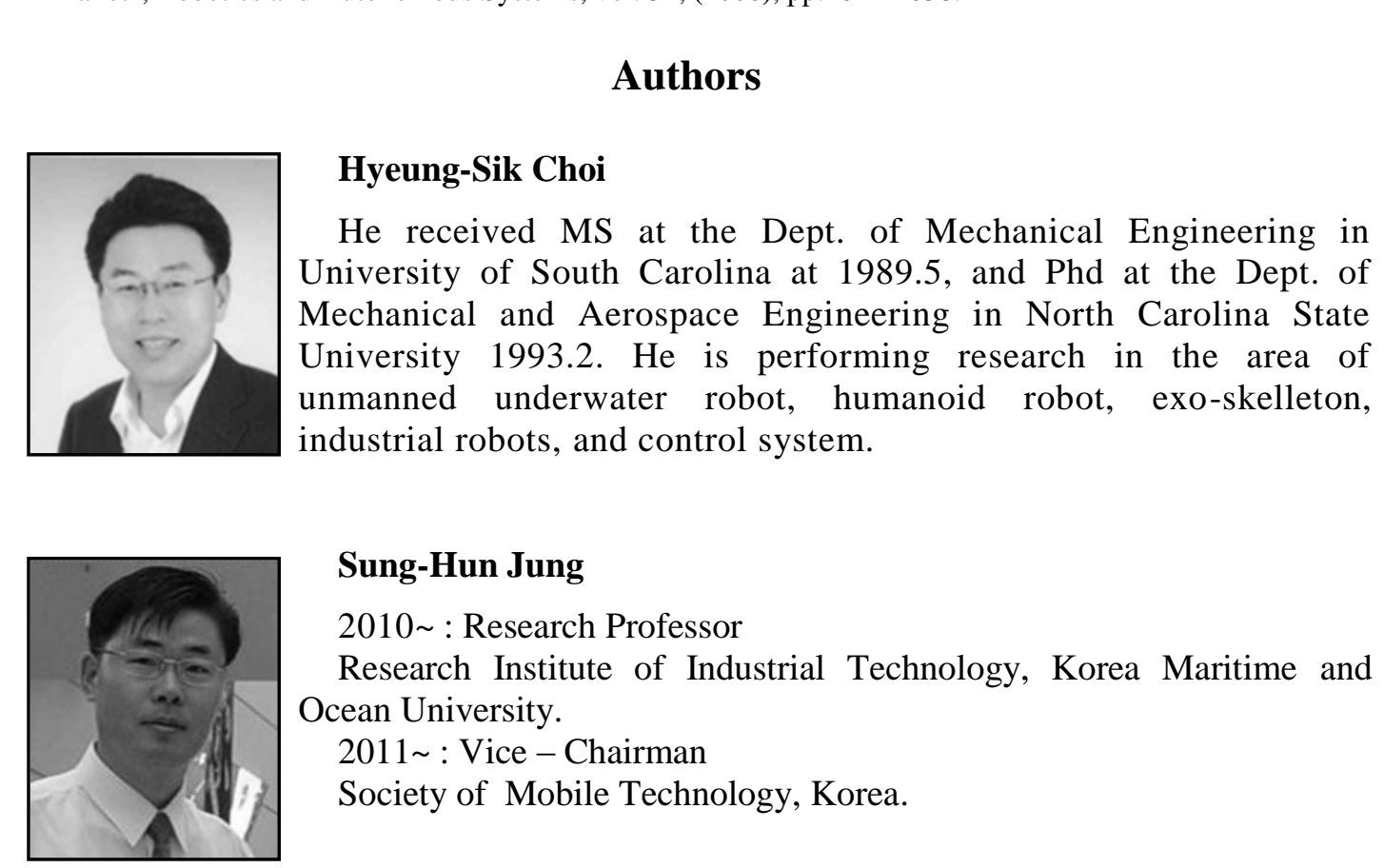

\section{Sung-Hun Jung}

2010 : Research Professor

Research Institute of Industrial Technology, Korea Maritime and Ocean University.

2011 : Vice - Chairman

Society of Mobile Technology, Korea. 


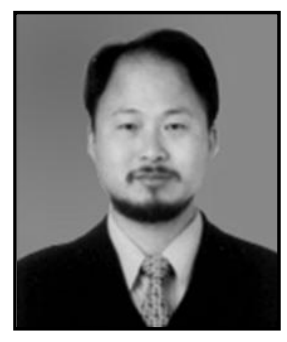

\section{Hwan-Seong Kim}

He received MS at the Dept. of Mechanical Engineering in Pukyong National University at 1993, and Phd in Kumamoto University at 1996 . He is performing research in the area of ocean system control and logistics equipment.

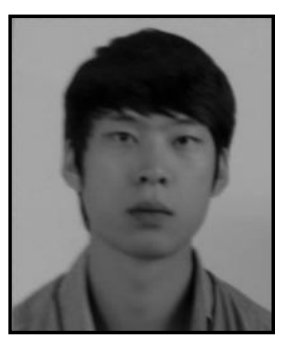

\section{Hee-Young Shin}

$\mathrm{He}$ received bachelor's degree at the Dept. of Mechanical Engineering in Korea Maritime and Ocean University at 2012.2, and the master's course at the Dept. of Mechlanical engineering in Korea Maritime and Ocean University. He is performing research in the area of unmanned underwater robot and eontrol system.

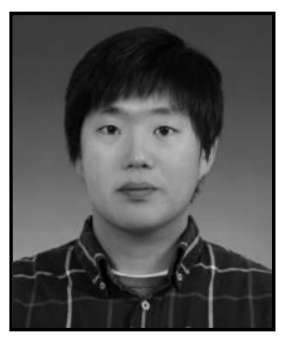

\section{Ji-Youn Oh}

He received bachelor's degree at the Dept. of Mechanical Engineering in Korea Maritime and Ocean University at 2013.2, and the master's course at the Dept. of Mechanical engineering in Korea Maritime and Ocean Qniversity. He is performing research in the area of unmanned

underwater robot and control system. 